\title{
Correlação do Equilíbrio e Qualidade de Vida de Idosos de um Centro Social em Belém-PA
}

\section{Correlation of the Balance and Quality of Life in The Elderly of a Social Center in Belém-PA}

\author{
Tânia Sanches Santos ${ }^{1}$ \\ Paloma da Silva Aranha de Carvalho ${ }^{2}$ \\ Márcio Clementino de Souza santos ${ }^{3}$ \\ George Alberto da Silva Dias ${ }^{4}$ \\ Cybelle Silva do Couto Coelho ${ }^{5}$ \\ Luciane Lobato Sobral ${ }^{6}$
}

\section{RESUMO}

Objetivo: Correlacionar o equilíbrio e a qualidade de vida dos idosos de um Centro Social em Belém - PA. Metodologia: O presente estudo foi observacional, descritivo e analítico, do tipo transversal. A amostra foi composta por 80 idosos, utilizando-se como instrumentos para a coleta dos dados, uma ficha de avaliação fisioterapêutica, a escalda de Tinetti, para avaliar o equilíbrio e o questionário SF-36, para avaliar a qualidade de vida. Utilizou-se os testes do Qui-quadrado, teste G (aderência) e correlação de Spearman para análise estatística. Resultados: Os resultados obtidos na amostra dos indivíduos idosos mostraram que ocorreu um predomínio do sexo feminino, com risco moderado de queda, com boa percepção da qualidade de vida para a saúde mental e pior percepção para os aspectos físicos. No entanto, todos os domínios da SF-36 correlacionaram positivamente com o equilíbrio. Conclusão: Diante dos resultados obtidos neste estudo, pode-se observar que os idosos apresentaram alterações no equilíbrio e na marcha e resultados satisfatórios nos domínios: saúde mental, aspectos sociais e vitalidade na escala de qualidade de vida, ensejando que a qualidade de vida está diretamente relacionada com o equilíbrio dos idosos, ou seja, quanto melhor o equilíbrio melhor é a percepção da qualidade de vida.

\section{DESCRITORES}

Envelhecimento. Marcha. Equilíbrio. Qualidade de vida.

\begin{abstract}
Objective: to correlate the balance and quality of life of the elderly of a Social Center in Belém - PA. Methodology: The present study was observational, descriptive and analytical, and cross-sectional. The sample consisted of 80 elderly, using a physiotherapeutic evaluation form for data collection. Tinetti's scalding and the SF-36 questionnaire were used to evaluate balance and quality of life. The Chi-square, G test (adherence) and Spearman correlation were used for statistical analysis. Results: The results obtained in the sample of elderly individuals showed that there was a predominance of females, with moderate risk of fall, with good perception of quality of life for mental health and worse perception for physical aspects. However, all domains of the SF-36 correlated positively with the balance. In view of the results obtained in this study, it can be observed that the elderly presented alterations in balance and gait, and satisfactory results in the domains of mental health, social aspects and vitality in the quality-of-life scale. Conclusion: Thus, it can be concluded that quality of life is directly related to the balance of the elderly, that is, the better the balance, the better the perception of quality of life.
\end{abstract}

\section{DESCRIPTORS}

Aging. March. Balance. Quality of life.

\footnotetext{
${ }^{1}$ Fisioterapeuta - Universidade da Amazônia (Unama). Pós-graduanda em Fisioterapia Pélvica - Uroginecologia Funcional (Faculdade Inspirar).

${ }^{2}$ Fisioterapeuta. Pós-graduanda em Traumato-ortopedia com ênfase em Terapia Manual e Postural (UNOPAR).

${ }^{3}$ Doutor em Doenças Tropicais (NMT/UFPA). Universidade do Estado do Pará (UEPA).

${ }^{4}$ Fisioterapeuta. Doutor em Doenças Tropicais (NMT/UFPA). Universidade do Estado do Pará (UEPA).

${ }^{5}$ Fisioterapeuta. Especialista em Traumato-ortopedia na Saúde e no Esporte (FINAMA). Mestranda em Neurociências e Comportamento (UFPA).

${ }^{6}$ Fisioterapeuta. Universidade do Estado do Pará. Doutora em Ciências do Movimento Humano.
} 
$\mathrm{N}$ Brasil, assim como em diversos países em desenvolvimento, o aumento no número da população idosa ocorre de forma rápida. Hoje, a população acima de 65 anos chega a $8,17 \%$, sendo estimado cerca de $13,44 \%$ de idosos até 2030 , segundo dados do Instituto Brasileiro de Geografia e Estatística (IBGE). Esse crescimento é acompanhado pelo aumento da expectativa de vida que, em 2016 , era de 75,7 anos, chegando a 78,64 anos em $2030^{1}$.

O envelhecimento é um processo natural, progressivo e dinâmico, no qual existem alterações morfofuncionais que tornam o organismo mais susceptível a agressões e a deformidades. À medida que uma pessoa envelhece, aumenta o risco de diversas doenças, dentre as quais aquelas que afetam o controle sensorial e motor dos pés ${ }^{1}$.

Sendo assim, o equilíbrio corporal apresenta declínios decorrentes do processo de envelhecimento. A prevalência de queixas de déficit de equilíbrio na população acima de 65 anos chega a $85 \%$. O equilíbrio envolve a recepção e a integração de estímulos sensoriais, o planejamento e a execução de movimentos para controlar o centro de gravidade sobre a base de suporte, realizado pelo sistema de controle postural, que integra informações do sistema vestibular, dos receptores visuais e do sistema somatossensorial ${ }^{2}$.

Pode-se dizer que envelhecer sem doença crônica é uma exceção, entretanto, ter a doença não significa necessariamente exclusão social, pois, se o idoso continuar ativo participando na sociedade e mantendo a sua autoestima, ele é considerado saudável pelos estudiosos. Dentre as doenças crônicas e as perdas apresentadas no processo natural do envelhecimento, destaca-se a diminuição do equilíbrio e a redução da marcha ${ }^{3}$.

A marcha é o conjunto de movimentos corporais repetidos, que devido ao envelhecimento, ocorre uma redução na velocidade e no comprimento da passada. O simples fato de andar requer atenção, força muscular, controle postural e coordenação. A marcha promove estabilidade, apoio e conservação de energia, que são preditores independentes da capacidade para realizar as atividades instrumentais da vida diária. Devido as alterações na marcha, o idoso pode ficar dependente, necessitando assim de dispositivos auxiliares para esta função, com o intuito de obter maior estabilidade ${ }^{4}$.

No âmbito da pessoa idosa, a qualidade de vida está relacionada à manutenção da autonomia, a qual pode ser percebida no desempenho das atividades diárias, nos idosos que mantêm sua capacidade funcional. A qualidade de vida supõe um elemento básico e interligado com todos os outros, que é a capacidade para realizar movimentos corporais de forma eficiente. Assim, para o autor, se a capacidade motora estiver limitada a qualidade de vida pode sofrer baixa considerável. Isso justifica a importância de propostas de intervenção que estimulem o uso ativo do corpo, pois é consenso entre os profissionais de saúde que a manutenção de um estilo de vida ativo é um fator determinante para o sucesso do processo de envelhecimento ${ }^{5}$.

Desse modo, avaliar as condições de vida do idoso é importante para o desenvolvimento de alternativas válidas de intervenção em programas de saúde e políticas sociais. Também é necessário o desenvolvimento de pesquisas e práticas de saúde que se 
preocupem com a qualidade de vida durante o processo envelhecimento. Uma forma possível de promover a saúde e a qualidade de vida é a utilização das práticas corporais em grupo. A modalidade grupal estimula o indivíduo participante a tornar-se o agente da própria mudança, além de propiciar um aprendizado interpessoal a partir do contato com o outro ${ }^{6}$. Dessa forma, o objetivo desse estudo foi correlacionar o equilíbrio e a qualidade de vida dos idosos de um Centro Social em Belém - PA.

\section{METODOLOGIA}

Aspectos éticos

A pesquisa teve início após a aprovação do Comitê de Ética em Pesquisa da Universidade da Amazônia (UNAMA) (Parecer número 3.313.974). Trata-se de um estudo observacional, descritivo e analítico, do tipo transversal seguindo as normas da Resolução n. 466/12, do Conselho Nacional de Saúde, relativa à pesquisa com seres humanos. Todos os participantes assinaram um termo de consentimento livre e esclarecido (TCLE).

\section{Amostra}

Participaram do estudo 80 idosos no período de abril a agosto de 2019 , sendo incluídos no estudo idosos de ambos os sexos e pertencentes a um Centro Social na Cidade de Belém-PA. Foram excluídos aqueles que não deambulavam ou utilizavam auxiliadores de marcha como muletas, andadores e cadeira de rodas, assim como, aqueles que eram hipertensos não controlados e que apresen- tavam alterações cognitivas. A amostragem foi por conveniência.

Procedimentos

Os dados foram coletados em formulário-padrão criado pelos pesquisadores, incluindo as seguintes variáveis: identificação, históricos de doença, dados antropométricos, avaliação da dor, bem como avaliação do estado mental como critério de exclusão. Posteriormente, foi avaliado o equilíbrio dos idosos por meio da escala de Tinetti, que detecta as alterações na locomoção, diagnostica e quantifica a gravidade do comprometimento. O teste é composto por duas escalas: de equilíbrio e de marcha. O resultado deste teste mostra o risco de queda do participante, onde valores abaixo de 19 pontos indicam alto risco de queda. Por fim, foi avaliado a qualidade de vida utilizando-se o questionário SF-36, que é um questionário genérico que avalia aspectos da qualidade de vida que estão diretamente relacionados a saúde do indivíduo e apresenta 8 domínios. O resultado deste teste demonstra que valores próximos de zero indicam pior percepção da qualidade de vida, e, aqueles próximos de 100 indicam o contrário.

Análise dos dados

Para análise estatística as variáveis categóricas foram apresentadas como frequências e as numéricas, por meio de medidas de tendência central. $\mathrm{Na}$ verificação da normalidade dos dados utilizou-se o teste D'Agostino-Pearson, onde evidenciou-se amostras não paramétricas. Foram utilizados o teste do Qui-quadrado, Teste G (Aderência) 
Tabela 1. Características da população idosa de um Centro Social em Belém, PA

\begin{tabular}{|c|c|c|c|}
\hline Variáveis & $n$ & $\%$ & p-valor \\
\hline \multicolumn{4}{|l|}{ Sexo } \\
\hline Masculino & 26 & 32,5 & \multirow{2}{*}{${ }^{a} 0,002^{*}$} \\
\hline Feminino & 54 & 67,5 & \\
\hline \multicolumn{4}{|l|}{ Idade (média 68,1 anos) } \\
\hline $60-69$ & 52 & 65,0 & \multirow{3}{*}{${ }^{b}<0,0001^{*}$} \\
\hline $70-79$ & 26 & 32,5 & \\
\hline 80 & 02 & 2,5 & \\
\hline \multicolumn{4}{|l|}{ Peso (kg) } \\
\hline $40-65,9$ & 32 & 40,0 & \multirow{3}{*}{${ }^{b}<0,0001^{*}$} \\
\hline $66-99,9$ & 46 & 57,5 & \\
\hline$\geq 100$ & 02 & 2,5 & \\
\hline \multicolumn{4}{|l|}{ Altura $(\mathrm{cm})$} \\
\hline $1,40-1,55$ & 30 & 37,5 & \multirow{3}{*}{${ }^{b}<0,0001^{*}$} \\
\hline $1,56-1,70$ & 47 & 58,75 & \\
\hline $1,71-1,95$ & 03 & 3,75 & \\
\hline \multicolumn{4}{|l|}{ Grau de Instrução } \\
\hline Analfabeto & 03 & 3,75 & \multirow{4}{*}{${ }^{b}<0,0001^{*}$} \\
\hline Ensino Fundamental & 37 & 46,25 & \\
\hline Ensino Médio & 32 & 40,0 & \\
\hline Superior & 08 & 10,0 & \\
\hline \multicolumn{4}{|l|}{ HDP } \\
\hline Diabetes & 10 & 12,5 & \multirow{6}{*}{${ }^{b} 0,0001^{*}$} \\
\hline HAS & 23 & 28,75 & \\
\hline Cardiopatia & 07 & 8,75 & \\
\hline Etilismo & 10 & 12,5 & \\
\hline Tabagismo & 03 & 3,75 & \\
\hline Outros & 27 & 33,75 & \\
\hline \multicolumn{4}{|l|}{ Dor } \\
\hline Cervical & 16 & 20,0 & \multirow{5}{*}{${ }^{b} 0,81$} \\
\hline Torácica & 16 & 20,0 & \\
\hline Lombar & 18 & 22,5 & \\
\hline Joelho & 12 & 15,0 & \\
\hline Sem Dor & 18 & 22,5 & \\
\hline
\end{tabular}

HDP: história da doença pregressa. (a) Teste do Qui-quadrado. (b) Teste G (Aderência). * Resultado estatisticamente significante, $\mathrm{p}<0,05$. 
e o de correlação de Spearman, adotando nível alfa de significância de $5 \%(p \leq 0,05)$. Todo o processamento estatístico foi realizado no software BioEstat 5.0.

\section{RESULTADOS}

Participaram desta pesquisa 80 idosos, sendo a maioria $(67,5 \%)$ do sexo feminino, com resultado significante $(p=0,002)$. Com relação à idade, a maioria $(65,0 \%)$ estava na faixa de 60 a 69 anos ( $p<0,0001)$, com peso entre 66 a 99,9 kg ( $n=57,5 \% ; p<0,001)$ e altura entre 1,56-1,70 cm ( $n=58,75 ; p, 0,0001)$. Para o grau de instrução, a maioria possuía ensino fundamental $(n=46,25 \% ; p<0,0001)$ e apresentava outras doenças pregressas $(n=33,75 \% ; p<0,0001)$. Porém, a variável dor não mostrou resultados significantes, conforme mostra a Tabela 1.
Com relação a percepção de saúde foi observado que o domínio com pior percepção foi o "limitação por aspectos físicos" e o de melhor percepção foi "saúde mental", evidenciando que os idosos são afetados com relação à qualidade de vida muito mais por aspectos físicos do que por saúde mental, conforme mostra a Tabela 2.

Na Tabela 3 foi possível observar pela média que o equilíbrio relacionado ao risco de queda dos pacientes foi de 20,38 pontos. Ou seja, eles apresentaram um risco moderado de quedas.

Por fim, buscando uma associação entre a qualidade de vida e o equilíbrio dos idosos, foi possível observar que todos os domínios do SF-36 tiveram relação significante com o risco de queda. Ou seja, quanto melhor é o equilíbrio dos idosos, melhor é a

Tabela 2. Dados em relação ao SF-36 da população idosa de um Centro Social em Belém, PA

\begin{tabular}{l|c}
\hline \multicolumn{1}{c|}{ SF-36 } & Média e Desvio Padrão \\
\hline Capacidade Funcional & $65,81 \pm 26,98$ \\
Limitação por Aspectos Físicos & $53,43 \pm 38,92$ \\
Dor & $69,25 \pm 24,71$ \\
Estado Geral de Saúde & $68,75 \pm 24,54$ \\
Vitalidade & $73,50 \pm 23,73$ \\
Aspectos Sociais & $77,18 \pm 25,83$ \\
Aspectos Emocionais & $62,49 \pm 40,87$ \\
Saúde Mental & $78,75 \pm 28,71$ \\
\hline
\end{tabular}

Tabela 3. Dados em relação ao teste de Tinetti da população idosa de um Centro Social em Belém, PA

\begin{tabular}{l|c}
\hline \multicolumn{1}{c|}{ Tinetti } & Média e Desvio Padrão \\
\hline Equilíbrio & $8,90 \pm 1,96$ \\
Marcha & $11,48 \pm 2,68$ \\
Total & $20,38 \pm 3,64$ \\
\hline
\end{tabular}


Tabela 4. Correlação entre a escala de Tinetti e qualidade de vida da população idosa de um Centro Social em Belém, PA

\begin{tabular}{l|c|c}
\hline \multicolumn{1}{c|}{ SF-36 } & $r$ & $p$-valor \\
\hline Capacidade Funcional & 0,2 & $0,01^{*}$ \\
Limitação por Aspectos Físicos & 0,4 & $0,0001^{*}$ \\
Dor & 0,2 & $0,01^{*}$ \\
Estado Geral de Saúde & 0,2 & $0,01^{*}$ \\
Vitalidade & 0,3 & $0,0006^{*}$ \\
Aspectos Sociais & 0,4 & $0,0002^{*}$ \\
Aspectos Emocionais & 0,4 & $<0,0001^{*}$ \\
Saúde Mental & 0,3 & $0,001^{*}$ \\
\hline
\end{tabular}

${ }^{*}$ Resultado estatisticamente significante. Teste de correlação de Spearman $(p<0,05)$.

percepção da qualidade de vida, conforme mostra a Tabela 4.

\section{DISCUSSÃO}

Os resultados obtidos na amostra de 80 indivíduos idosos mostraram que em relação às características gerais dos voluntários, ocorreu um predomínio do sexo feminino e com ensino fundamental, o que corrobora com a pesquisa de $\mathrm{Morsch}^{35}$, onde $37,7 \%$ dos idosos possuíam até o ensino fundamental, a maior porcentagem do grau de instrução como a deste estudo. A doença e a complicação mais apresentada foi a hipertensão arterial representando $28,75 \%$, no estudo de Giaquini $^{36}$ a hipertensão arterial também foi a variável que obteve a maior porcentagem, cerca de $40,6 \%$.

Em relação a qualidade de vida, os resultados obtidos mostram que estão de acordo com a literatura e cada domínio está dentro dos padrões de normalidade. O domínio saúde mental, foi calculado a partir da média do estado geral de saúde, vitalidade, função social, aspectos emocionais e saúde mental ${ }^{7}$. Este domínio apresentou média escore mais alto com 78,75 de pontos, estando de acordo com a literatura do estudo de Amancio et al. ${ }^{37}$, onde relata média escore de 66,60 , a partir disso perceber-se que o estado psicológico dos idosos dessa pesquisa está satisfatório e dentro dos padrões de normalidade.

O domínio limitação por aspectos físicos avalia o quanto estas limitações podem interferir na vida diária do indivíduo ${ }^{8}$. Os idosos participantes deste estudo tiveram uma média escore de 53,43, o índice mais baixo em relação aos outros domínios, porém este valor continua entre os padrões de normalidade e corrobora com o estudo de Amancio et al. ${ }^{37}$, onde a média escore foi de 58,37.

O estado geral de saúde está relacionado como o idoso considera sua saúde de uma forma global ${ }^{9} \mathrm{e}$ os idosos dessa pesquisa tiveram um índice bastante satisfatório neste domínio. Assim, os idosos da presente pesquisa apresentam um estado geral de saúde excelente, com a média escore de 68,75, acima da média escore geral do questionário SF-36 que é 50 .

Os aspectos sociais avaliam o quanto a sua saúde física ou problemas emocionais interferiram em suas atividades sociais ${ }^{10,11}$. Verificou-se que este foi o segundo domínio com a pontuação mais alta, com a média escore de 77,18 que condiz com a literatura, média 
escore de 75. Ou seja, na maioria dos idosos a sua saúde física ou problemas emocionais não interferiram em suas atividades sociais. Pode-se justificar está média alta, devido às intervenções e programas de saúde que o centro social oferece, promovendo a socialização entre os idosos.

No domínio dor, observou-se que o resultado obtido foi relevante, com média escore de 69,25 , tendo os idosos participantes dessa pesquisa apresentado na avaliação cerca de $22,5 \%$ de dor na região lombar, em decorrência de más posturas e alterações fisiológicas do envelhecimento, mostrando que os valores da avaliação fisioterapêutica e do domínio dor do questionário da qualidade de vida estão correlacionados.

As alterações no padrão de marcha ocorrem com o envelhecimento tanto nos fatores fisiológicos quanto emocionais, podendo ser percebidas pelas alterações motoras ${ }^{25}$. Nos resultados do teste de Tinetti, verificou-se que a variável marcha apresentou bons resultados com a média de 11,48 , condizente com a literatura que relata 11,65 no estudo de Piovesan et al. ${ }^{33}$.

Porém, o resultado da variável equilíbrio apresentou um índice baixo com média de 8,90, não condizente com a literatura, onde no estudo de Bushatsky et al. ${ }^{34}$ relataram uma média de 14. Percebe-se que alguns

\section{REFERÊNCIAS}

1. Machado, Á. S. et al. Efeitos da manipulação da sensibilidade plantar sobre o controle da postura ereta em adultos jovens e idosos. Revista Brasileira de Reumatologia. 2017; 57(1):30-36

2. Isang EYL, Liamputtong $P$, Pierson J. The views of order Chinese people in Melbourne about their quality of life. Ageing \& Society 2014; 51-74

3. Sarmento WE, Sobreir, FMM, Oliveira AMB. Avaliação do Equilíbrio e da Mobilidade de Idosos após um Programa de Escola de Posturas. Rev Bras Ciên da Saúde. 2014; 18(1):27-32. idosos sofrem uma grande alteração em seu equilíbrio podendo assim interferir em suas atividades diárias. O envelhecimento fisiológico leva a alterações posturais, de equilíbrio, prejudicando a velocidade da marcha e largura dos passos, aumentando a base de suporte.

A pontuação total do teste de Tinetti, apresentou uma média de 20,38 neste estudo, estando dentro dos padrões de normalidade, comparado ao estudo de Piovesan et al..$^{33}$ com a média 24. Verificou-se que os idosos participantes desse estudo, apresentaram alterações no equilíbrio e que está correlacionado com a qualidade de vida, ou seja, quanto maior o valor obtido em Tinetti, maior é o valor obtido na qualidade de vida.

\section{CONCLUSÃO}

Diante dos resultados obtidos neste estudo, pode-se observar que os idosos apresentaram alterações no equilíbrio e na marcha, e resultados satisfatórios nos domínios saúde mental, aspectos sociais e vitalidade, pela escala de qualidade de vida. As variáveis do equilíbrio e da marcha estão correlacionadas com a qualidade de vida, onde obteve resultados estatisticamente significante, mostrando que o equilíbrio e a marcha influenciam na qualidade de vida dos idosos, ou seja, quanto melhor for o equilíbrio do idoso, melhor é a sua percepção da qualidade de vida.

4. Santo, D. Características da marcha de idosos considerando a atividade física e o sexo. 12 páginas. Estudo de caso. Fisioterapia. Universidade do Estado de Santa Catarina CEFID. 2008.

5. Steter A.M. et al. Importância da avaliação do equilíbrio e marcha do idoso. J Health Sci Inst. 2014; 32(1):43-47.

6. Karuka AH. Et al. Análise da concordância entre instrumentos de avaliação do equilíbrio corporal em idosos. Rev Bras de Fisioterapia. 2011; 15(6):460-466.

7. Almeida VC, Meira SS, Gomes FV, Souza MV, Santos VC, Anjos KF. Qualidade de vida em idosos que sofreram quedas. Rev. APS. 2014; 17(4):530-536. 
8. Teixeira CS, Link DM, Ribeiro JK, Costa VP, Mota CB. Aspectos biomecânicos do caminhar em idosos. In: XVII Jornada Acadêmica Integrada. Anais Acadêmicos da Universidade Federal de Santa Maria (UFSM): Santa Maria; 2014

9. Noronha FRD et al. Equilíbrio estático de mulheres idosas submetidas a um programa de atividade física. Rev. Bras. Geriatr. Gerontol. 2015; 18(4):735-742.

10. Abreu SSE, Caldas CP. Velocidade de marcha, equilíbrio e idade: um estudo correlacional entre idosas praticantes e idosas não praticantes de um programa de exercícios terapêuticos. Rev. Bras Fisioter. 2008;12(4):324-330.

11. Anderson MIP. Quedas seguidas de fratura e hospitalização em idosos: frequência, circunstâncias e fatores de risco (tese de Doutorado). Rio de Janeiro: UERJ; 2013.

12. Antes $\mathrm{D}$. Mortalidade por queda em idosos. 10 páginas. Estudo de série temporal. Fisioterapia. Universidade Federal de Santa Catarina. 2015

13. Aruka AH, Silva JAMG, Navega MT. Análise da concordância entre instrumentos de avaliação do equilíbrio corporal em idosos. Rev. Bras Fisioter. 2013;15(6):460-466.

14. Bertolini AB et al. Marcha no processo de envelhecimento: alterações, avaliação e treinamento. Revista Uningá. 2015; 45:52-55.

15. Bispo N. Alterações no equilíbrio e na marcha e o risco de quedas em idosos. 6 páginas. Estudo de caso. Fisioterapia. Universidade Norte do Paraná. 2006.

16. Bittar RSM, Pedalini MEB, Bottino MA, Formigoni IG. Síndrome do desequilíbrio no idoso. Pró-fono, Rev Atualização Científica 2014; 14(1):119-128.

17. Foroni P. Fatores de risco e proteção associados ao declínio cognitivo no envelhecimento. 10 páginas. Artigo de revisão. Medicina. Faculdade de Medicina de Ribeirão Preto. 2012.

18. Gawryszewski VP. A importância das quedas no mesmo nível entre idosos no Estado de São Paulo. Rev Assoc Med Bras. 2013; 56(2):162-167.

19. Instituto Brasileiro de Geografia e Estatística - IBGE. Síntese de indicadores sociais. Estudos e pesquisas. Informação demográfica e socioeconômica. Rio de Janeiro: IBGE; 2014; 5:369.

20. Lojudice DC et al. Equilíbrio e marcha de idosos residentes em instituições asilares do município de Catanduva, SP. Rev. Bras. Geriatr. Gerontol. 2008; 11(2):181-189.

21. Maia $B C$, et al. Consequências das quedas em idosos vivendo na comunidade: revisão sistemática. Rev. Bras. Geriatr Gerontol. 2014; 14(2):381-393.

22. Melo MCD, et al. A educação em saúde como agente promotor de qualidade de vida para o idoso. Ciência \& Saúde Coletiva. 2009; 14(1):579-1586

23. Noronha NCB, Pelegrini AC, Grohs IB. Alterações no equilíbrio e na marcha e o risco de quedas em idosos. Cient Cienc Biol Saúde. 2006; 8(1):5-9.
24. Paula FL. Envelhecimento e quedas de idosos. $1^{\text {a }}$ ed. Rio de Janeiro: Apicuri; 2013.

25. Santos SI, et al. Desempenho da marcha de idosos praticantes de psicomotricidade. Rev Bras de Enfermagem. 2014; 67(4):617-622.

26. Silva IA, et al. Efeito de um protocolo de Facilitação Neuromuscular Proprioceptiva (FNP) no equilíbrio postural de idosas. Fisioterapia e Pesquisa. 2017; 24(1):62-67.

27. Silva J. Análise da alteração do equilíbrio, da marcha e o risco de queda em idosos participantes de um programa de fisioterapia. 8 páginas. Estudo de caso. Fisioterapia. Universidade Estadual Paulista. 2014.

28. Szydloski TP, et al. Marcha e equilíbrio em idosos institucionalizados: avaliação pré- e pós- exercícios físicos. Rev Kairós Gerontologia. 2015; 18(2):327-338.

29. Toldrá RC, et al. Promoção da saúde e da qualidade de vida com idosos por meio de práticas corporais. O Mundo da Saúde. 2014; 38(2):159-168.

30. Uchida JEF, Borges SM. Quedas em idosos institucionalizados. Rev Kairós. 2013; 16(3):83-94.

31. Vasconcelos NN, et al. Análise dos parâmetros da marcha e do equilíbrio dos idosos após exercícios aeróbicos e terapêuticos. Arq. Ciênc. Saúde UNIPAR. 2016; 20(1):1923.

32. Xavier FMF, Ferraz MPT, Marc N, et al. A definição dos idosos de qualidade de vida. Rev. Bras Psiquiatr. 2013 25(1):31-39.

33. Piovesan AC, Soares ES, et al. Avaliação do Teste de Tinetti e Mini-Exame do Estado Mental em idosas moradoras da comunidade Roberto Binatto, Santa Maria (RS). Revista Kairós Gerontologia. 2015;18(1):341-352.

34. Bushatsky A, Alves LC, Duarte YAO, Lebrão ML. Fatores associados às alterações de equilíbrio em idosos residentes no município de São Paulo em 2006: evidências do Estudo Saúde, Bem-Estar e Envelhecimento (SABE). Rev. Bras. Epidemiol. 2018; 21 (Suppl 2): E180016

35. Morsch P, Myskiw M, Myskiw JC. A problematização da queda e a identificação dos fatores de risco na narrativa de idosos. Ciência e Saúde Coletiva. 2016; 21(11):35653574.

36. Giaquini $F$, Lini EV, Doring $M$. Prevalência de dificuldade de locomoção em idosos institucionalizados. Acta Fisiatr. $2017 ; 24(1): 1-6$.

37. Amancio TG, Oliveira MLC, Amancio VS. Fatores que interferem na condição de vulnerabilidade do idoso. Rev Bras Geriatr Gerontol. 2019; 22(2):e180159.

\section{CORRESPONDÊNCIA}

Tânia Sanches Santos

Fisioterapeuta - Universidade da Amazônia (Unama)

Pós-graduanda em Fisioterapia Pélvica -

Uroginecologia Funcional (Faculdade Inspirar)

E-mail: taniasanches.fisio@gmail.com 